\title{
Temperature dependence of the resistive switching-related currents in ultra-thin high-k based MOSFETs
}

\author{
Albert Crespo-Yepes, ${ }^{\text {a) }}$ Javier Martin-Martinez, Rosana Rodriguez, Montse Nafria, and \\ Xavier Aymerich \\ Departament d'Enginyeria Electrònica, Universitat Autònoma de Barcelona (UAB) 08193, Bellaterra, Spain
}

(Received 30 July 2012; accepted 10 January 2013; published 31 January 2013)

\begin{abstract}
In this work, the temperature dependence of the resistive switching phenomenon in metal-oxidesemiconductor field-effect-transistor (MOSFETs) with an ultra-thin Hf-based high-k dielectric is studied through analysis of the gate and drain currents for the two dielectric conductivity states. These two different conductive states of the resistive switching have been associated with the dielectric breakdown (BD) and dielectric BD reversibility (R), respectively, and are related to the creation of a $\mathrm{BD}$ path through the dielectric that can be understood as a conductive filament. The results of the temperature dependence of the post-BD gate current are in agreement with those obtained from the study of the injected charge to recovery, which is a useful parameter with which to analyze the switch from the high to low conductivity state. The drain current in the MOSFETs for the two conductivity states, for different locations of the BD path along the channel (close to the source and close to the drain), and at several temperatures has also been studied. The results contribute to a better understanding of the resistive switching phenomenon in ultra-thin gate dielectrics. This contribution could be useful for the developing of models to describe BD reversibility. (C) 2013 American Vacuum Society. [http://dx.doi.org/10.1116/1.4789518]
\end{abstract}

\section{INTRODUCTION}

One of the most important failure mechanisms in metaloxide-semiconductor (MOS) technologies is the gate dielectric breakdown (BD), ${ }^{1}$ a state where the insulator conductivity changes (switches) from a low conduction state to a larger one with a current difference of several orders of magnitude. In deep submicron complementary metal-oxide-semiconductor (CMOS) technologies, high-k materials have been introduced to overcome the leakage problems associated with the scaling of $\mathrm{SiO}_{2}$ as the gate dielectric, and hence, a complete analysis of the dielectric BD on these high-k materials is needed. ${ }^{2}$ Recently, it has been reported that dielectric BD in ultra-thin Hf-based high-k gate dielectric stacks can be a reversible phenomenon, with two interchangeable conductivity states in the dielectric that can be reached by applying the correct stress scheme and a current limited stress during the $\mathrm{BD}$ transient. $^{3,4}$ Moreover, it has been shown that when the dielectric switches back from the high conductivity state to the low conductivity state the device performance is partially restored and the circuit lifetime is increased. ${ }^{5}$

A change between different conduction states in the dielectric material also characterizes the resistive switching (RS) phenomenon usually observed in metal-insulator-metal/metalinsulator-semiconductor (MIM/MIS) capacitors with a thick insulator (several tenths of a nanometer), which is being widely investigated as the operation principle of resistive random-access-memory (RRAM) nonvolatile memories. This principle can be understood as the formation of a conductive filament that can be closed again, i.e., by destruction of the filament. Recently, the similarities between the RS and the $\mathrm{BD} /$ recovery mechanisms have been reported, and the authors

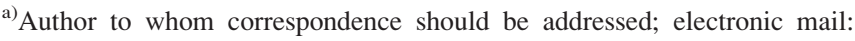
Albert.Crespo@uab.cat
}

suggest that the mechanisms can actually have the same origin. ${ }^{6}$ Therefore, the exhaustive characterization of the RS phenomenon in ultra-thin high-k dielectrics has a twofold interest: one, for the development of memory devices based on this phenomenon and the other, from a reliability point of view, for the development of models that can help to evaluate the device and circuit performance and reliability. In this work, the temperature dependence of the resistive switching phenomenon in such ultra-thin films is studied through the analysis of the gate and drain currents in a MOSFET when the dielectric is at the low or high conductivity states. A recently presented stress methodology is used, which allows better control and characterization of the BD recovery process. $^{7}$

\section{SAMPLES AND EXPERIMENT SET-UP}

The samples used in this work were pMOSFETs with a fully-silicated (FUSI) gate electrode and dielectric stack equivalent-oxide-thickness $(\mathrm{EOT})=1.9 \mathrm{~nm}$ formed by an HfSiON film (2.9 nm physical thickness) on top of a $1.2 \mathrm{~nm}-$ thick $\mathrm{SiO}_{2}$ interfacial layer. Transistors with varying widths (ranging from 1 to $0.15 \mu \mathrm{m}$ ) and having the same channel length $(0.35 \mu \mathrm{m})$ were used. To characterize the BD reversibility, the stress-measurement scheme depicted in Fig. 1 was adopted. After the measurement of the fresh electrical transistor characteristics $\left(\mathrm{I}_{\mathrm{G}}-\mathrm{V}_{\mathrm{GS}}\right.$ and $\left.\mathrm{I}_{\mathrm{D}^{-}} \mathrm{V}_{\mathrm{DS}}\right)$, the samples were subjected to a stress sequence, which consisted of a currentlimited ramp voltage stress (CL-RVS) to provoke the dielectric $\mathrm{BD}$ (i.e., change from the low to the high conductivity state, or BD-state), plus a stair-case voltage stress (SCVS) without current limitation to recover the dielectric (i.e., switch back to the low conductivity state, R-state). An SCVS was chosen to switch to the low-conductivity state instead of the standard RVS because SCVS allows for the evaluation of the injected charge to recovery, $Q_{R}$, which has been shown to be 


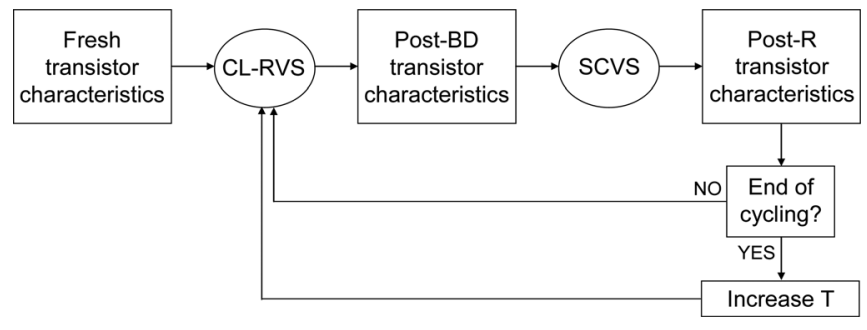

FIG. 1. Stress-measurement sequence designed to analyze the dielectric BD reversibility. The compliance of the measurement equipment was used to limit the current during current-limited stress (CL-RVS).

a useful parameter to characterize the BD recovery. ${ }^{7}$ Both stresses were applied to the gate with the rest of the terminals grounded. During the SCVS the gate voltage was decreased, starting from $-0.6 \mathrm{~V}$, by approximately $-0.1 \mathrm{~V}$ every $\sim 150 \mathrm{~s}$ until a maximum of $-1.3 \mathrm{~V}$ at $1200 \mathrm{~s}$. After each CL-RVS and SCVS, the transistor characteristics were again recorded. In each sample, the iterative sequence in Fig. 1 was repeated for many cycles. The temperature of the measurements was increased in the same sample every 20 or 40 cycles, during which the temperature was maintained constant, and upon completion the temperature was increased by $50^{\circ} \mathrm{C}$ starting from $25^{\circ} \mathrm{C}$ and increasing up to $175^{\circ} \mathrm{C}$ (Fig. 1).

\section{RS-RELATED GATE CURRENTS}

Figure 2 shows the gate currents in a pMOSFET with $\mathrm{W} / \mathrm{L}=1 \mu \mathrm{m} / 0.35 \mu \mathrm{m}$ and at $25^{\circ} \mathrm{C}$ during the CL-RVS [Fig. 2(a)] and SCVS [Fig. 2(b)], for the 1st, 3rd, and 10th cycles of the measurement sequence (Fig. 1). In Fig. 2(a), I FRESH corresponds to the gate current measured during the first CLRVS (continuous line). At $\sim-5 \mathrm{~V}$, a sudden increase of the
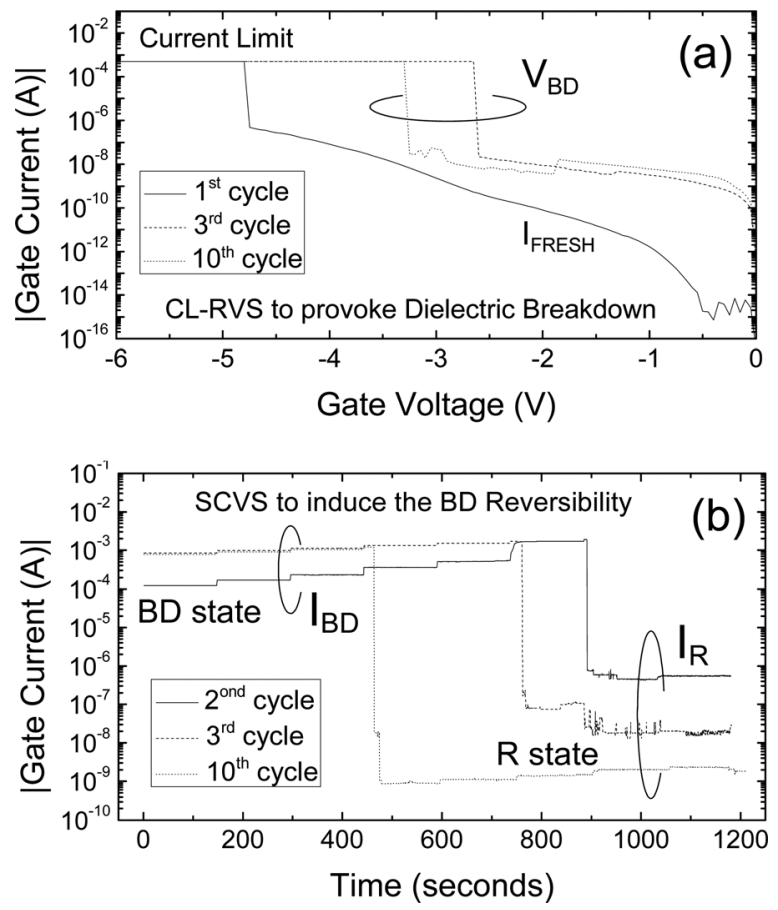

FIG. 2. Gate current recorded (a) during the CL-RVS necessary to reach the $\mathrm{BD}$ state and (b) during the SCVS necessary to provoke the BD recovery (R-state). gate current up to the current limit $(500 \mu \mathrm{A}$ in this case) is observed, which indicates that the dielectric BD has taken place and a conductive filament (BD path) has been created in the dielectric (forming phase), leading the dielectric to the $\mathrm{BD}$ state. After BD, the gate current measured during the following SCVS [continuous line in Fig. 2(b)] is, as expected, greater $\left(\mathrm{I}_{\mathrm{BD}}\right)$ than in the fresh device. However, at a given time, a fast current drop of several orders of magnitude is observed $\left(\mathrm{I}_{\mathrm{R}}\right)$, which indicates $\mathrm{BD}$ recovery; i.e., the BD path which had been "opened" in the previous CL-RVS has been closed, reaching the low conductivity state (R-state).

In successive cycles, the $\mathrm{BD}$ path is again opened [at $\mathrm{V}_{\mathrm{BD}}$ during the CL-RVS, Fig. 2(a)] and closed during the SCVS [Fig. 2(b)]. This change between stet high and a low conductivity state in the dielectric is observed for many iterations and is qualitatively repetitive from sample to sample. ${ }^{6}$

In order to analyze the effect of temperature in the switching process, the sample temperature has been raised during cycling, and $\mathrm{Q}_{\mathrm{R}}$ was calculated in each iteration from the $\mathrm{I}_{\mathrm{BD}}$ and time values until the first recovery event during the SCVS of each cycle [Fig. 2(b)]. ${ }^{7}$ Figure 3 shows the cumulative probability function of $\mathrm{Q}_{\mathrm{R}}$ distributions measured in the same sample when the temperature was increased from 25 to $175^{\circ} \mathrm{C}$ (with $50^{\circ} \mathrm{C}$ increments) every 20 cycles. Concerning the steeper region of the distributions, $\mathrm{Q}_{\mathrm{R}}$ dispersion increases when the temperature is increased. On the other hand, a clear decrease of the mean value of $Q_{R}$ with temperature is observed; i.e., the injected charge needed to change from the high to the low conductivity state decreases with temperature. A direct relation between $\mathrm{Q}_{\mathrm{R}}$ and the degradation level of the $\mathrm{BD}$ path (i.e., the difficulty of the BD event) has been established (the more difficult the BD event, the larger the $\mathrm{Q}_{\mathrm{R}}$ ), ${ }^{7}$ so that the results in Fig. 3 seem to suggest that the damage (loss of insulator properties of the dielectric) created in the dielectric during the current-limited BD transient decreases with temperature. If a filamentary character of the $\mathrm{BD}$ path is considered, a lower $\mathrm{I}_{\mathrm{BD}}$ measured when increasing the temperature could be caused by the higher resistance of the dielectric. Since $\mathrm{Q}_{\mathrm{R}}$ depends on $\mathrm{I}_{\mathrm{BD}}$ and time-to-recovery [Fig. 2(b)], the temperature dependence of these magnitudes has been analyzed showing that as the temperature increases, not only does

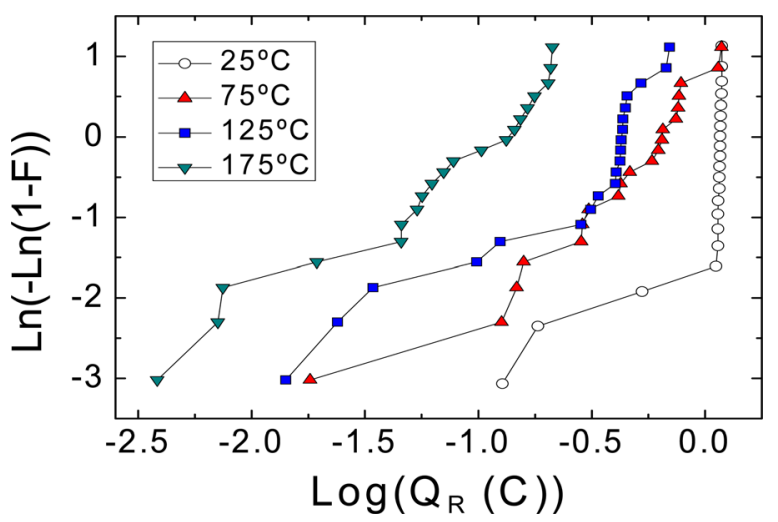

FIG. 3. (Color online) Statistical distributions of the $\mathrm{Q}_{\mathrm{R}}$ measured during cycling on the same sample. The "F" function represents the cumulative probability function of $\mathrm{Q}_{\mathrm{R}}$. Distributions are organized by temperature, which was increased by $50^{\circ} \mathrm{C}$ every 20 cycles in the same sample. 
TABLE I. Mean time to BD recovery during the SCVS as a function of temperature.

\begin{tabular}{lllll}
\hline \hline Temperature $\left({ }^{\circ} \mathrm{C}\right)$ & 25 & 75 & 125 & 175 \\
Mean time to BD recovery (s) & 584 & 446 & 335 & 211 \\
\hline \hline
\end{tabular}

the $\mathrm{I}_{\mathrm{BD}}$ current decrease but also the time needed to reach the $\mathrm{R}$ state. Note that this time decreases linearly with temperature (Table I). Therefore, increasing the temperature favors the switching to the low conductivity state.

The temperature dependence on the RS-related gate currents in the device, namely, the post-BD and the post- $\mathrm{R} \mathrm{I}_{\mathrm{G}^{-}}$ $\mathrm{V}_{\mathrm{G}}$ characteristics obtained after each CL-RVS and SCVS, respectively, has been analyzed for the different temperatures. The $I_{G}-V_{G}$ curves are symmetric for both voltage polarities and for the two dielectric conductivity states. Figure 4 shows the $\mathrm{I}_{\mathrm{G}}$ evolution with cycling measured at $\mathrm{V}_{\mathrm{G}}=-0.5 \mathrm{~V}$ after the BD transient (post-BD currents, red circles) and after the $\mathrm{BD}$ recovery (post-R currents, open circles). Figure 4 shows that, though the current through the BD path is approximately constant within each of the isothermal cycles sets, the post$\mathrm{BD} \mathrm{I}_{\mathrm{G}}$ decreases with temperature. This could be interpreted as a smaller amount of damage in the dielectric during the $\mathrm{BD}$ transient with increasing temperature or as a temperature dependent post-BD gate stack conduction mechanism. Contrarily, at the R-state, no temperature dependence is observed on the post-R $\mathrm{I}_{\mathrm{G}}$. Further studies are needed to explain these temperature dependences of the post-BD and post-R gate currents.

\section{DRAIN CURRENTS DURING SWITCHING}

The effect that the gate stack BD event and BD recovery at different temperatures have upon the drain transistors' electrical characteristics has been analyzed. To perform a more complete analysis, different locations of the conductive $\mathrm{BD}$ path along the channel were considered, which paths were determined using the method presented in Ref. 8. Figure 5(a) shows the fresh and post-R $I_{D}-V_{D S}$ characteristics measured in two samples with the BD path located at the source or

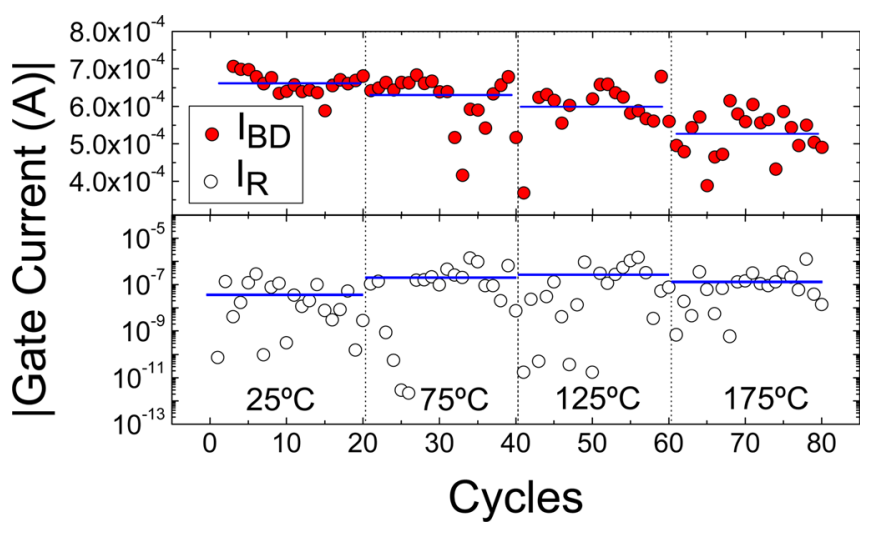

FIG. 4. (Color online) $\mathrm{I}_{\mathrm{G}}$ evolution with cycling after $\mathrm{BD}$ transient (colored circles) and after BD recovery (open circles). Every 20 cycles the temperature was increased by $50{ }^{\circ} \mathrm{C}$, starting from $25^{\circ} \mathrm{C}$ until $175^{\circ} \mathrm{C}$ is reached. Blue lines indicate the mean values at each temperature.
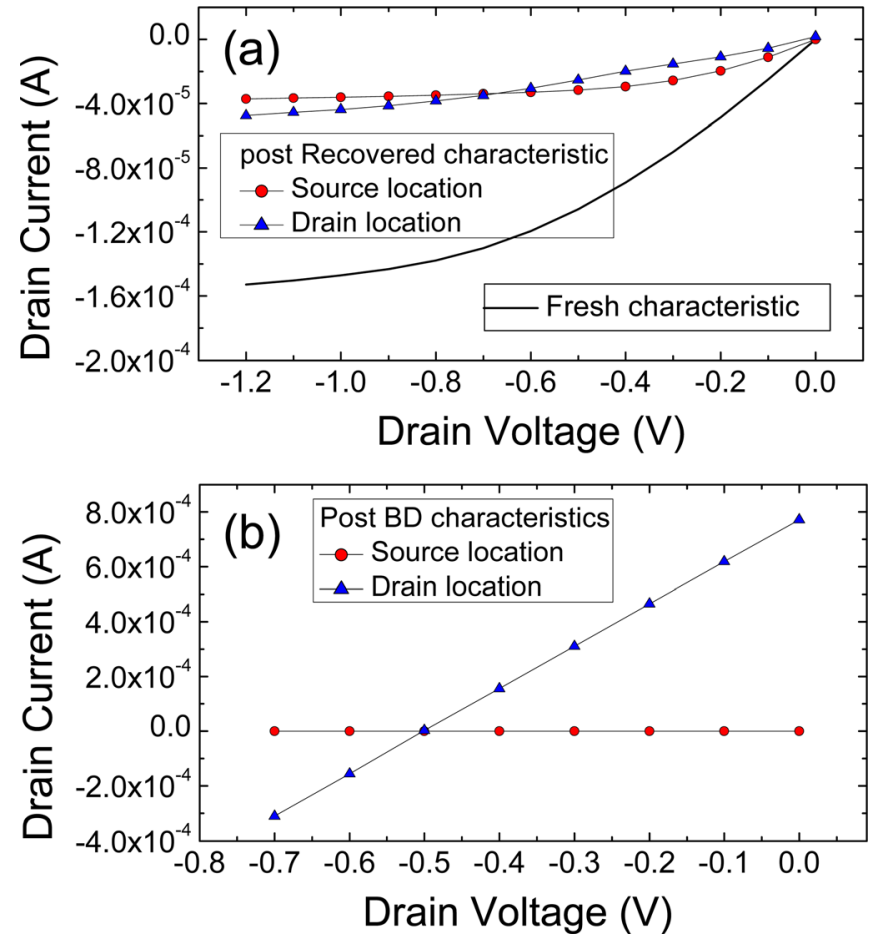

FIG. 5. (Color online) Drain current versus drain voltage measured for (a) fresh and recovered devices, with $\mathrm{BD}$ paths located close to drain or source and (b) after inducing the BD in the same samples.

drain. Independent of the $\mathrm{BD}$ path location, after the $\mathrm{BD}$ recovery the $I_{D}-V_{D S}$ curves resemble those of a fresh device, which indicates that the device can still operate as a MOSFET but with a large reduction of the drain current. ${ }^{6}$ Contrarily, when the characteristics are measured during the BD state, the transistor characteristics are completely distorted [Fig. 5(b)]. However, the $I_{D}-V_{D S}$ shape depends on the BD path location along the channel; when the $\mathrm{BD}$ path is located close to the drain (triangles) the drain current mainly flows between drain and gate through the BD path. As a consequence, a purely resistive behavior in the $I_{D}-V_{D S}$ characteristic is observed, which should be attributed to the resistance offered by the BD path (note the zero-crossing of the drain current at $\mathrm{V}_{\mathrm{G}}=\mathrm{V}_{\mathrm{D}}$, i.e., when no voltage is applied to the path). When the BD path is located close to the source (circles), a very low drain current is measured $(\approx 10 \mathrm{pA})$. In this case, the current through the BD path flows between the gate and source without contributing to the drain current. Furthermore, the low current measured at the drain indicates that there is no channel current contribution when the gate stack is in the BD state. Please note that the measured behavior does not depend on the channel width. In summary, in the BD state, the transistor loses its functionality not only due to the high current through the BD path but also because the inversion layer is not created in the transistor channel. However, for both BD path locations, the $\mathrm{I}_{\mathrm{D}}-\mathrm{V}_{\mathrm{DS}}$ characteristic can be partially restored when the stack is switched to the $\mathrm{R}$ state.

The drain current has been studied at different device temperatures and biasing conditions. Figure 6 shows the post-BD drain current measured at $V_{D}=-0.7 \mathrm{~V}$ for several gate voltages and temperatures during the cycling for $\mathrm{BD}$ path 

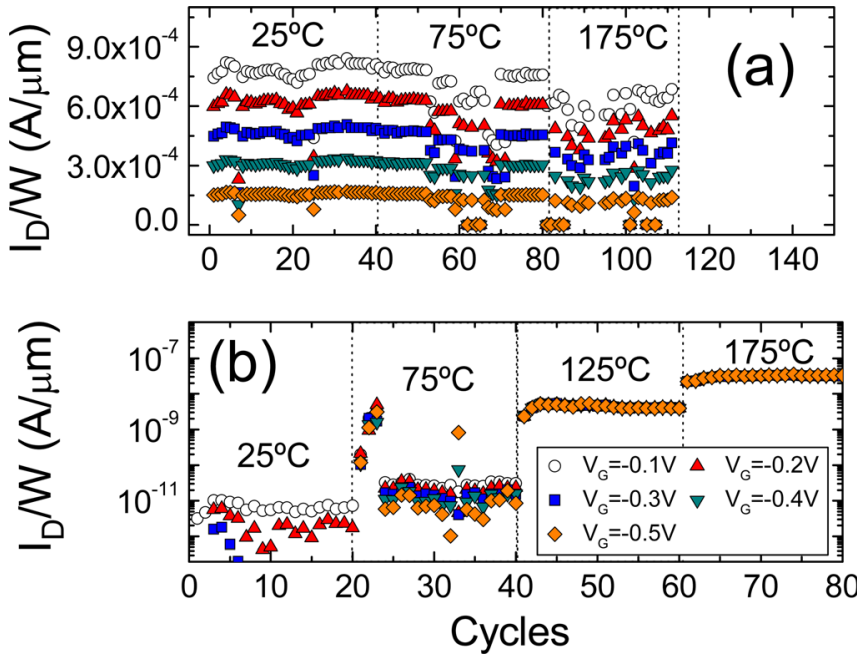

FIG. 6. (Color online) Drain current measured at different temperatures in devices after $\mathrm{BD}$, when the conductive path is located close to drain (a) or source (b).

locations at the drain [Fig. 6(a)] and source [Fig. 6(b)]. For the case of $\mathrm{BD}$ at the drain, clearly the drain current increases when the gate voltage decreases (in absolute value) in the entire studied temperature range. This can be explained by the larger contribution of the $\mathrm{BD}$ gate current toward the drain current, because the voltage drop at the BD-path increases when the gate voltage decreases. At high temperatures the drain current is lower, due to the decrease of the BD path conductivity at high temperatures, as previously observed in Fig. 4. If the BD path is located at the source [Fig. 6(b)], the drain current is very small [Fig. 5(b)] and increases with temperature. Moreover, gate voltage dependence is only observed at the lower temperatures. For all the temperatures, the drain currents are very low, and therefore, we can conclude that the inversion layer of the MOSFET is never created when the BD path is at the source and at the high-conductivity state.

Figure 7 shows the drain current (normalized to the transistor width) obtained in MOSFETs during the cycling after the $\mathrm{BD}$ recovery where the $\mathrm{BD}$ paths are located at the drain [Fig. 7(a)] and source [Fig. 7(b)]. In this case, no meaningful differences are observed in the drain currents for the two BD path locations and at all temperatures (and drain currents around $30-60 \mu \mathrm{A}$ are measured). Therefore, after the BD recovery, the conduction through the BD path has no relevant influence on the transistor characteristics. Moreover, the inversion layer, which could not be formed at the BD state, is always partially restored when the sample switches to the $\mathrm{R}$ state so that the drain current is controlled by the channel current. Note that when the stack is at the $\mathrm{R}$ state and $\mathrm{T}$ is kept constant a progressive increase of drain current is observed (Fig. 7), contrary to the observed behavior when the BD path is open (Fig. 6). Additional studies should be done to completely understand this dependence.

From these results, it can be concluded that $\mathrm{BD}$ is a reversible phenomenon in the typical temperature range of the device operation in a chip, and that the MOSFET performance is partially restored when the BD path is switched off.
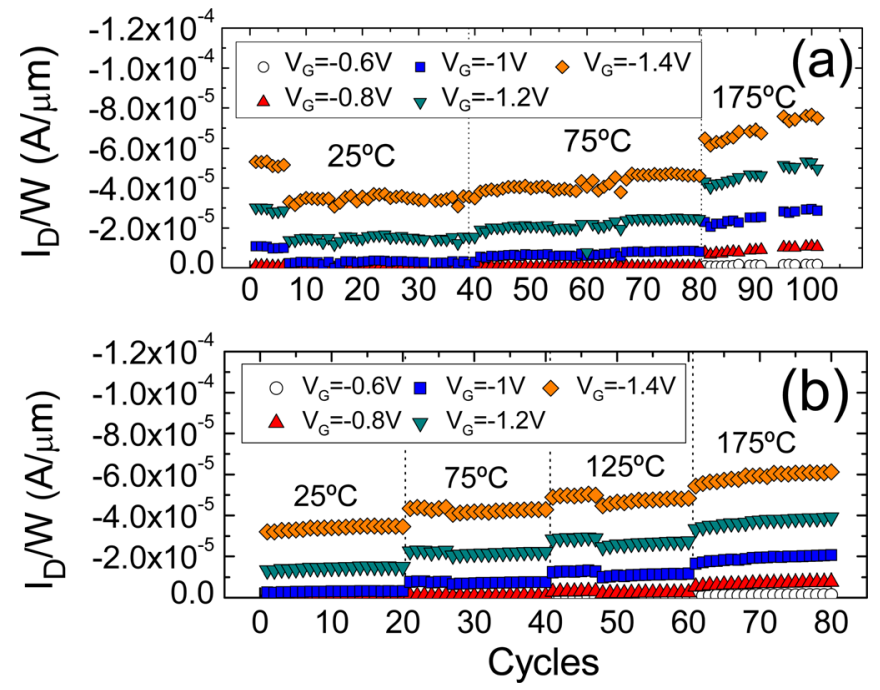

FIG. 7. (Color online) Drain current (normalized to the transistor width) measured at different temperatures in devices after the BD recovery when the conductive path is located at the drain (a) or source (b). Similar currents are measured in both cases which stet that after recovery the BD path location has no relevant influence in the transistor electrical characteristics.

\section{CONCLUSIONS}

In this work, the resistive switching phenomenon in MOSFETs with ultrathin Hf based high-k dielectrics have been studied at different temperatures. The two different conductive states of the resistive switching have been associated to the BD and BD recovery of the dielectric. The results show that at high temperature the BD recovery takes place after lower values of the injected charge to recovery, though with a larger dispersion because both the post-BD current and the time-to-recovery decrease with temperature. The dependence of the RS-related currents (gate and drain) on temperature has been analyzed. At the BD state, the gate current through the high-k stack decreases with temperature whereas, after BD recovery, the gate current does not depend on temperature. The drain current in MOSFETs for the dielectric BD and $\mathrm{R}$ states and for different locations of the BD path along the channel (source and drain) and at several temperatures have been studied. After BD, the MOSFET loses its functionality because of the high current through the BD path (when BD is at drain) and/or the impossibility of forming the inversion layer (BD is at source). However, after BD recovery, the gate current contribution to the drain current is negligible and also the inversion layer can be formed so that the MOSFET functionality is restored, though with reduced performance. This behavior has been observed in the $25-175^{\circ} \mathrm{C}$ temperature range, which leads us to conclude that $\mathrm{BD}$ reversibility in MOSFETs can be produced even at the high temperatures that can be reached in a chip. The results reported here represent new features of gate stack conductivity switching, which could be very useful for development of models to describe the BD reversibility.

\section{ACKNOWLEDGMENTS}

This work has been partially supported by the Spanish MICINN (TEC2010-16126 and TEC2010-10021-E) and the Generalitat de Catalunya (2009SGR-783). 
${ }^{1}$ E. Y. Wu and J. Suñe, Microelectron. Reliab. 45, 1809 (2005).

${ }^{2}$ R. Degraeve, M. Aoulaiche, B. Kaczer, P. Roussel, T. Kauerauf, S. Sahhaf, and G. Groeseneken, Proceedings of the 15th International Symposium on the Physical and Failure Analysis of Integrated Circuits (2008), pp. 239-244.

${ }^{3}$ W. H. Liu, K. L. Pey, X. Wu, N. Raghavan, A. Padovani, L. Larcher, L. Vandelli, M. Bosman, and T. Kauerauf, Appl. Phys. Lett. 99, 232909 (2011).

${ }^{4}$ A. Crespo-Yepes, J. Martin-Martinez, R. Rodriguez, M. Nafria, and X. Ayemrich, Microelectron. Reliab. 49, 1024 (2009).
${ }^{5}$ A. Crespo-Yepes, J. Martin-Martinez, A. Rothschild, R. Rodriguez, M. Nafria, and X. Aymerich, IEEE Electron Device Lett. 31, 543 (2010).

${ }^{6}$ A. Crespo-Yepes, J. Martin-Martinez, A. Rothschield, R. Rodriguez, M. Nafria, and X. Aymerich, Solid-State Electron. 65-66, 157 (2011).

${ }^{7}$ A. Crespo-Yepes, J. Martin-Martinez, A. Rothschield, R. Rodriguez, M. Nafria, and X. Aymerich, IEEE Trans. Device Mater. Reliab. 11, 126 (2011).

${ }^{8}$ R. Degraeve, B. Kaczer, A. De Keersgieter, and G. Groeseneken, International Reliability Physics Symposium (2001), pp. 360-366. 